Théologiques

Théologiques

\title{
Amour pur et union en dépit de l'absence dans Laylî u Majnûn de Nizâmî
}

\section{Jad Hatem}

Volume 16, numéro 2, 2008

L'individualisation de la relation religieuse

URI : https://id.erudit.org/iderudit/001715ar

DOI : https://doi.org/10.7202/001715ar

Aller au sommaire du numéro

Éditeur(s)

Faculté de théologie et de sciences des religions, Université de Montréal

ISSN

1188-7109 (imprimé)

1492-1413 (numérique)

Découvrir la revue

Citer cet article

Hatem, J. (2008). Amour pur et union en dépit de l'absence dans Laylî u Majnûn de Nizâmî. Théologiques, 16(2), 67-85. https://doi.org/10.7202/001715ar
Résumé de l'article

Après avoir acquis son titre de référence des amants, la figure de Majnûn Laylâ a été mobilisée par la mystique pour exprimer l'amour pur, à savoir l'union à Dieu en dépit de l'absence. À l'instar du Majnûn de la légende arabe, qui s'est enfoncé sans recours et jusqu'à la folie dans l'amour désespéré, celui de la mystique voue à Dieu une dilection sans bénéfice. Dans le roman que Nizâmî lui consacre, deux rêves, l'un du personnage principal, l'autre d'un compagnon, viennent rétablir l'équilibre. Dans le premier, de teneur subjective, l'amant est consacré roi, ce qui est de nature à concevoir un accomplissement au sein même de l'absence; dans le second, la réunion des amants dans la mort redéfinit le paradis comme le lieu de l'union. 


\title{
Amour pur et union en dépit de l'absence dans Laylî u Majnûn de Nizâmî
}

\author{
Jad HATEM* \\ Philosophie et mystique comparée \\ Université Saint-Joseph
}

Quand, en 1188, Nizâmî reprend à son compte la légende de Majnûn dans Laylî u Majnûn, il n'entend pas seulement l'orner des fastes dont sont capables son génie et la poésie persane. C'est en penseur frotté d'idées mystiques qu'il aborde la légende arabe à laquelle il est le premier à conférer le statut de roman courtois. Bien que le poète ne fût pas reçu officiellement dans un ordre, son œuvre porte la marque de l'influence soufie. Je m'efforcerai de dégager les éléments mystiques du roman en marquant sa signification ascétique et le sens de l'amour qu'il véhicule, amour d'identification à l'Aimé en dépit de son absence. Toutefois, le trait singulier du roman, par contraste avec la légende arabe dont il s'inspire, tient au fait qu'une union dans la présence est affirmée, mais à travers le rêve, comme une réalisation dans l'infini, en tout cas par-delà le monde.

\section{Amour, fantasme et réalité}

De manière significative, Nizâmî voit en la passion amoureuse l'ivresse qu'on ne peut réprimer, le signe de la plus grande passivité. Qays s'y noie avant même de savoir ce que c'est, ce qui revient à dire, d'une part, qu'il ignore qu'à ce mot correspond ce sentiment et, d'autre part, qu'il est submergé par la vague déferlante avant même de réaliser ce qui lui arrive et qu'il faille enfin lui conférer le mot de 'ishq (Hatem 2000). L'amour acquiert sur lui un tel empire qu'il lui conquiert immédiatement station

Jad Hatem est professeur de philosophie, de littérature et de sciences des religions à l'Université Saint-Joseph (Beyrouth). Ses recherches actuelles portent sur la mystique féminine. Il a récemment publié (2008) La Poésie de l'extase amoureuse. Shakespeare et Louise Labé, Paris, Orizons. 
d'ivresse qui est perte de soi. Nizâmî veut que la jeune Laylâ (condisciple de Qays) éprouve la même morsure. C'est pourquoi il exprime l'événement en termes d'inter-possession: ils boivent simultanément à la coupe enivrante. Puisqu'ils sont deux, chacun ne se perd que pour retrouver l'autre. De quelle nature est cette inter-possession ${ }^{1}$ ? Deux modalités en sont repérables. L'une se décline dans la visibilité et l'épaisseur du monde où la coïncidence se cherche dans la conspatialité et l'étreinte charnelle. L'autre, qui n'est pas objet de quête ou de revendication, se donne entièrement dans l'étreinte spirituelle qui fait fi du visage de l'aimée, étreinte qui domine à ce stade, d'autant que Nizâmî précise que le monde extérieur et la salle de classe s'effacent de la conscience des amants submergés par l'extase. Mais il appartient à l'essence de l'amour d'être animé de deux formes de désir, dès lors que l'inter-possession vient à faire défaut selon ses deux modalités: si l'amant ne s'estime pas aimé d'une affection égale à la sienne, s'allume en lui le désir de l'étreinte par le sentiment. Si maintenant l'amour est partagé, il aspire à se réaliser également dans la visibilité du monde, car on demande d'un amour qui illumine le cœur qu'il aille également illuminer le monde. Mais dans ce cas, le risque est que la proximité extramondaine s'inverse en éloignement dans le monde.

C'est le leitmotiv du roman que rien ne dure sous le soleil. Le monde est frappé au coin de l'éphémère et la vie n'est qu'un conte, un éclair dans la nuit, une illusion. Il en résulte que le désir est, par nature, contrarié par le destin. N'est-ce pas laisser soupçonner que l'amour de Majnûn et de Laylâ exemplifie, en dépit de son unicité, une réalité universelle? Certes, même ici des motifs qui relèvent de la psychologie peuvent être allégués. Le jeune Qays prend conscience du caractère incertain de tout ce qui advient dans le monde en considérant la beauté miraculeuse de l'aimée et la convoitise qu'elle suscite déjà alentour. Il était donc prévisible que, la voyant, Ibn Salâm s'en éprendrait et demanderait sa main. Toutefois, ces impulsions relèvent de la contingence (celui-ci plutôt que celui-là). C'est la structure générale de l'être qui imprime aux relations le caractère de l'impermanence. Est-ce à dire que l'intériorité est préservée de tout fléchissement ? L'expérience le démentirait. En première approximation, je crois l'idée de Nizâmî voisine : elle invite à conclure que toute variation affective dépend d'influences extérieures. Et c'est précisément parce qu'il s'est définitivement fermé à de telles influences que Qays met sa passion à l'abri de toute intrusion, lors même qu'elle occupe tout l'espace que l'extériorité revendi-

1. Sur cette notion, voir Hatem (2008). 
quait comme sien: en effet, la carapace de la monade craque et révèle l'à-vif de son âme - ouverture qui permet d' "affectiver " tout l'être, autrement dit, de modifier en intensités «laylifiées " les relations extensives sociales et naturelles. Parce que son ensauvagement est proprement démondanéisation et que sa folie affiche l'identification de la pure immanence à l'amour pur, la souplesse et la fluidité de sa vie affective se trouvent disqualifiées au profit d'une fidélité sans faille parmi les choses périssables. C'est cette rigidité que Majnûn appelle fatalité. L'ouverture ne laisse rien pénétrer. En réalité, le mot ne convient que pour la manifestation du sentiment. Le mouvement est plutôt d'expansion. En revanche, c'est la fixation de l'identité sociale qui se trouve annulée. Que le jeune Qays attire l'opprobre sur la famille de Laylâ, qu'il se perde lui-même en la perdant, qu'en lui se dissolve la distinction du bien et du mal ou que l'impression le hante qu'il a été comme effacé du livre de la vie, qu'il finisse par préférer la compagnie des bêtes, qu'il semble un démon en forme humaine, tout cela traduit la liquéfaction de son identité sociale qui passe entièrement dans le regard multiplié d'autrui - allant de l'oubli pur et simple à la permutation du nom: de Qays à Majnûn.

Mais qu'en est-il lorsque la fatalité alléguée se retourne sur elle-même dans le mouvement d'appropriation de l'affect? La passion amoureuse s'apparaîtra comme inaltérable, inentamable, et donc perpétuelle. C'est pourquoi, en deuxième approximation, le lecteur apprend de Nizâmî que le temps passe, tandis que la véritable passion amoureuse persiste. La vie de ce monde n'est, pour la plus grande part, qu'une succession d'illusions et de déceptions. Mais la véritable passion amoureuse est réelle, si bien que les flammes qui la consument brûlent à jamais, sans commencement ni fin. Soudain, une créature, l'amour humain, est élevé à un rang supranaturel, voire divin, si bien qu'il maintient dans l'orbe de l'être la chose évanescente et la plus chère. Déjà libéré du monde, il revêt une condition métaphysique. Nûrî se trompait donc en tenant la passion pour un état transitoire. Il eût été mieux inspiré de définir en ces termes l'aspiration amoureuse et nostalgique dénommée shawq, appelée à disparaître aussitôt qu'on atteint l'aimé (Ibn Qayyim al-Jawziyya 1956, 29). Il est à remarquer que cet état n'exige pas la réciprocité (à moins que la poésie de l'amant ne soit elle-même réponse de l'aimée). Majnûn ne distingue pas se consumer et briller, car le combustible ne lui paraît pas devoir faire défaut. De fait, le combustible relève du divin. Majnûn s'est en quelque sorte quintessencié ou plutôt resubstantié par une sorte de réduction phénoménologique à l'être pur qui 
s'avère être passion amoureuse: "De mon être l'amour est l'essence, Il est ce feu dont je suis le brandon» $(224$, v. 11$)$.

Dans le cas de Majnûn comme chez maints spirituels, cette réduction emprunte la forme de l'ascèse : jeûnes et macérations (privation des plaisirs que procure le monde) au profit de la purification, voire renoncement aux grâces (privation des joies que procureraient Laylâ et Dieu). Que dans cet ordre le topos corresponde à la pensée intime du poète persan, le lecteur s'en avise qui étend son enquête jusque dans l'essai qui fait partie de la Pentalogie nizâmîenne, à savoir Makbzan ul-asrâr. Y domine l'appel adressé à l'homme (qui est poussière) de renoncer, moyennant les austérités, au monde, lequel, éphémère, ne mérite que mépris :

Puisque nous devons retourner à la poussière, pourquoi passer au-dessus de cette terre? Ne piétine personne parce que le destin en a piétiné plus d'un comme toi. Nul n'a vécu en ce monde à jamais, nul n'a détenu ce décret de la vie éternelle. Ne marche pas sur cette épine, lève-toi, protège-toi contre elle. Ce séjour impermanent qui est le tien est un lieu de peur; pourquoi rester en un tel endroit? Ce monde est transitoire, ne le considère pas éternel. (Nizâmî 1987, 124-125)

Rien qui ne consonne avec le roman courtois, mais aussi rien qui coïncide avec les formes supérieures de la mystique de la passion amoureuse ou de l'identité. Pour confirmer ces banalités que reproduisent tous les traités de spiritualité, Nizâmî confie à la religion le soin d'assurer le salut: «La nature n'a pas les moyens de t'amener au salut. Dans la cage de l'oiseau, il n'y a que peu de vie [...]. Se détourner des désirs est la souveraineté, y renoncer totalement est le pouvoir prophétique [...]. Fuis pour ton salut vers le sanctuaire de la religion, afin d'être libéré de soucis au jour de la résurrection » (105). Ailleurs, il invite son lecteur à recevoir en son âme les lois religieuses (166). Certes Majnûn fait montre de fanatisme dans l'abandon du monde qui dépasse les injonctions somme toutes mesurées du Makhzan ul-asrâr. Il y a comme un degré juste au-dessus du renoncement aux valeurs du monde (zuhd fîl-dunyâ), qui consiste en la sortie de la civilité et l'entrée dans un anachorétisme sauvage dont l'islam n'offre pas beaucoup d'exemples. Mais cette supériorité d'un degré ne change rien quant à l'essentiel, à savoir que le roman demeure fidèle à la ligne de l'ascèse. Les illustrateurs l'ont bien vu qui ont représenté le personnage en fakir, demeurant néanmoins en deçà du texte qui le décrit, lors de la visite que lui fait l'amateur de poésie Sallâm, nu de la tête aux pieds, en conformité avec le paradigme du renonçant absolu de type indien. Un autre argument permet 
de retenir le substrat intellectuel du roman dans la littérature ascétique, et par là dans le registre des grandeurs moyennes: manquent au roman les percutantes sentences d'identification qui ont assuré la transition vers la reprise en mystique du thème majnûnien. La remémoration constante de Laylâ n'atteint pas la conscience d'extinction, elle figure seulement l'expulsion de ce qui n'est pas elle, voire de l'égo de Majnûn, en faveur de l'apprésentation de l'aimée, ce que produit exactement l'exercice spirituel du $d h i k r$ : l'avènement de la passion qui a investi la maison l'a expulsé de son site. Il n'y existe plus. Seule y réside l'aimée, ce pour quoi il peut dire que sa vie est mesurée par elle (Leylî u Majnûn, 224, v. 13-17). C'est qu'il a déjà prononcé la sentence d'identification: "Je suis toi, un seul cœur pour deux personnes » $(215$, v. 10$)$.

On voit pourtant qu'on ne saurait réduire la folie de Majnûn à un exercice d'ascèse quand il est expression de la passion. Quand bien même cellelà est théoriquement fondée sans rapport à celle-ci (puisque le monde perd comme tel toute valeur), elle lui doit d'être son effet. On ne se trompe sans doute pas en tenant qu'il est dans l'intention de Nizâmî de rendre l'ascèse de Majnûn analogue à celle des spirituels. Mais on est en droit de douter que son personnage voie en son aimée Dieu lui-même ou un simple moyen dont il se sert pour atteindre Dieu, thèmes traités ostensiblement par Jâmî.

\section{Le rêve royal}

S'il y a une mystique au sens supérieur, chez Nizâmî, elle me paraît devoir être repérée dans la signification transcendante de cette réalité que met en évidence la réduction phénoménologico-ascétique. Nizâmî a choisi le langage onirique pour la suggérer. Le premier rêve a notre héros pour auteur.

Après avoir reconnu que les planètes demeuraient sourdes à son appel pour la bonne raison qu'elles ne sont pas animées, et partagent avec lui la condition de sujets, Majnûn se tourne vers le Créateur, Celui à qui tout est soumis. Redevable à Dieu pour la vie, il lui devra de précipiter l'heure du trépas. La prière prend une tournure inattendue: "Ce soir, je me tiens devant toi certes comme quelqu'un qui vit et respire, mais aussi comme celui dont l'âme même est morte. Seule ta miséricorde peut me sauver maintenant; seule ta grâce peut m'épargner la damnation éternelle; ta compassion seule pourrait inverser mes ténèbres en lumière et ma nuit en jour. » Le cœur serein, Majnûn s'endort et fait le rêve suivant: devant lui, un arbre surgit soudain de la terre. Il le voit croître à grande vitesse jusqu'à ce qu'il le surplombe. Le suivant du regard alors qu'il monte jusqu'aux cieux, il 
remarque un oiseau perché sur une des branches les plus élevées. Quelque chose scintille dans le bec de l'oiseau qui prend son vol et plane au-dessus de Majnûn avant de laisser choir l'objet scintillant. C'est un bijou et il tombe directement sur la tête de Majnûn. Il demeure là, pareil au brillant joyau d'un diadème (tâj). Majnûn se réveille transporté de joie (180, v. 5-10).

Le cadre du rêve indique assez que le souci de Majnûn est avant tout religieux : savoir si, en dépit de son idée fixe qui lui a fait demander, lors du pèlerinage à la Mecque, de persister dans sa passion (comme Shiblî dans sa folie; voir Lory 2001, 78), Dieu le préservera de la damnation. Notons que même ici il ne réclame pas la grâce d'échapper à l'impitoyable règne sur lui de la Passion ou même d'obtenir l'aimée, projet que les planètes se sont montrées incapables de favoriser. Il reste à s'interroger sur le sens de cette damnation éternelle. Elle porte la marque tangible de la réprobation divine. Elle signifie davantage que même dans l'au-delà les amants seront séparés. De là l'appel à la compassion qui pourrait inverser les ténèbres en lumière et la nuit en jour. Que Laylâ cesse pour lui d'être nuit obscure pour se changer en éclat visible.

Le rêve prend son départ à la fois dans cette demande et dans l'image d'une âme défunte. C'est en effet de l'âme elle-même que surgit l'arbre qui s'élève brusquement. D'emblée un signe de vie, inflation qui dément l'âme rabougrie. Mais quoi de l'âme ou dans l'âme a pu germer? Le contexte oblige à privilégier en priorité le regard tourné vers les hauteurs, regard déçu par les planètes, prière qui semble atteindre son destinataire divin. Le Livre saint compare la proclamation de la bonne parole de l'unicité de Dieu à un bel arbre dont la racine est ferme tandis que sa ramure est dans le ciel (Coran 14,24). La métaphore se change en image visible lors de l'ascension mystique de Bistâmî qui, doté d'ailes, s'élève de station en station jusqu'à «l'arbre de l'unicité » (ahadiyyat; Sarrâj 1960, 464). L'arbre se présente comme un puissant symbole d'union: il réunit la terre et le ciel (axis mundi), les deux sexes à titre d'équivalent du Mercure en alchimie (voir Jung 1971, 334) — l'arbre pouvant même être dédoublé en plante porteuse de fruits solaires et plante porteuse de fruits lunaires (Pernety 1758, 40). Il signifie donc l'intégration et la totalisation. Celui que décrit l'alchimiste Abû al-Qâsim Muhammad $(1923,23)$ porte même quatre couleurs, symbole de complétude². Celui qu'imagine Ibn 'Arabî (1961) s'entoure de quatre oiseaux. Une précision s'impose: l'arbre symbolise la vie et

2. Ce n'est pas un hasard si ces couleurs correspondent à celles que la tradition attribue aux quatre tempéraments. 
s'il renvoie plus précisément à l'âme (ou l'Anthropos; Jung 1971, 449), il allégorise un processus. Jung précise : il représente "le Soi comme phénomène de croissance» (329). La célérité avec laquelle celui de Majnûn se dresse marque un accomplissement qui ne se présente pas comme effectif et se contente de s'annoncer dans le rêve oraculaire. Il faut donc convenir que le simple arbre ne saurait symboliser l'achèvement de l'opus. Pour qu'il le fasse, il lui faut adjoindre une autre figure archétypale: ses branches pourraient par exemple se nouer comme un androgyne, ou virer en disque solaire. Or parmi les compléments essentiels qui permettent de marquer, comme fait ou comme projet, la totalisation, on rencontre le motif de l'oiseau. Jung écrit: "L'arbre avec l'oiseau représente l'œuvre et son accomplissement » (414). Telle me paraît la clef psychologique du rêve de Majnûn. Couronné par la phase de l'esprit, l'opus se réfléchit dans une conscience élargie et immortelle. L'Islam confirmerait à sa manière l'intuition alchimique par cela que le Coran $(17,13)$ compare le sort et l'œuvre de chaque homme à un oiseau. Par le contact du haut et du bas, la vie est communiquée à l'âme. Renaissance qui est investiture, car la couronne symbolise aussi l'accomplissement (348). Le joyau qui se détache de l'oiseau figure la pierre philosophale, la propre âme de Majnûn en tant que portée à incandescence, autrement dit, brillante de l'éclat même du désir essentiel.

Voici Majnûn roi et, comme le révélait un rêve déjà mentionné, pardonné par Dieu et devenu post-mortem la référence des amants (AlQushayrî 1996, 322). La détresse l'abandonne. Son existence reçoit du rêve confirmation. Toutefois, pour ne plus plier sous le joug impérial de la démence qui sacrifie le sens transcendant après avoir immolé le pôle mondain, l'élan intentionnel de l'amour reste indéterminé. Autrement dit, rien ne semble être dit de retrouvailles avec l'aimée. Le rêve est tel et le quotidien demeure livré à l'ascèse, mais désormais la continence est comparable au jeûne qui, selon Mullâ Sadrâ $(1966,369)$, est «paradis de feu ». Un retour à l'immanence de l'amour pur paraît être suggéré par l'ultime séquence du rêve. Au mouvement d'ascension fait pendant la descente du joyau. Que si ce joyau typifie Laylâ, Majnûn sera condamné à célébrer des noces avec sa seule anima (puisque le joyau est le produit de l'âme), ou, en termes nizâmiens, entre l'âme et l'intellect ${ }^{3}$. Que s'il ne la typifie pourtant guère, il peut espérer étreindre la personne réelle, ce que le deuxième rêve entend révéler.

3. «L'Eau de la Vie est l'âme avec l'intellect» (Les Sept Portraits, 41). 


\section{Le rêve paradisiaque}

En première analyse, Majnûn, uni à Laylâ dans la mort, semble également l'obtenir dans l'au-delà selon le rêve de Ziyâd ${ }^{4}$, une sorte de double du fou qui s'interrogeait sur le destin posthume des âmes jumelées, à savoir si elles sont confinées dans les ténèbres de la tombe ou ont trouvé place dans le Paradis. Le Majnûn du Diwân arabe n'osait entrevoir l'existence paradisiaque tout en soutenant vigoureusement la pérennité de l'amour. Sa position est donc mitigée, et non moins originale. En cette heureuse issue, l'analogie avec la voie ascétique peut se poursuivre jusqu'à sa conclusion : au renonçant aussi, celui qui a su traverser avec succès les épreuves et endurer sans faillir le moratoire imposé au désir, il sera donné d'atteindre le port de la permanence et de la jouissance, par une sorte de compensation méritée. Et si le renoncement est doublé de passion ou se fonde sur elle, c'est Dieu même et non un simple lieu de béatitude qui sera rejoint. Mais ce n'est toujours qu'une analogie, car même ici rien n'identifie Laylâ à Dieu. L'idée est ailleurs: dans la conquête du sens de l'être comme passion. Le monarque du 'ishq est affranchie de la matière et de ses concupiscences. On a vu que le 'ishq est l'essence de son être. Majnûn a conjuré l'errance causée par le désir d'une femme et a transcendé les sentiments honteux qui faisaient de lui un serf du 'ishq. La consumation de son âme charnelle l'anéantit si peu qu'elle ne fait que le reconduire à la substance vive de l'être. De quoi rappeler la thèse avicennienne de la présence de l'éros en la nature entière et de l'ascension érotique vers ce qui est digne d'amour, et surtout la théorie hallâjienne du 'ish q comme attribut incréé de Dieu et cause de la création (voir Hatem 2006 et 2005). Et de fait, le 'ishq qu'atteint Majnûn a pris le caractère de la pérennité divine, car il ne diminue guère et n'est pas illusoire. Hâfiz disait : "La couleur de la passion existait alors que les deux mondes étaient absents" (Dîvan-e Hâfez, $\mathrm{n}^{\circ}$ 16). Dieu n'est le primum desideratum que dans un deuxième moment, car il lui appartient d'être le premier désirant. C'est d'être désir et de se désirer qu'il suscite tous les désirs.

Il s'ensuit qu'en deuxième analyse, le contenu du rêve doit être rabattu sur l'expérience de l'éros substantiel, ce 'ishq éternel que le poète distingue du stérile et éphémère jeu des passions juvéniles. Ce contenu relève nécessairement du désir quand bien même il se voudrait une simple révélation. Le couple réuni allégorise une parousie de l'être comme flamboiement du

4. Si cette conclusion du roman n'est pas de la plume de Nizâmî, l'interpolation ne dessert pas son dessein car elle donne figure à l'idée de la supranaturalité de l'amour. 
désir et appel à imitation. Certes le Majnûn du Diwân arabe pouvait être requis pour confirmer la survie du feu passionnel au pourrissement des corps, mais c'était pour perpétuer dans la tombe le tribut de sang lacrymal versé de son vivant.

L'épisode du rêve de Ziyâd tire d'ailleurs son efficacité de l'exemplarité de Majnûn dont l'intronisation comme seigneur dans le royaume de la passion amoureuse invite à un vasselage d'un type particulier, par mimétisme. Rûzbehân, autre contemporain exact de Nizâmî, note que Majnûn devient le miroir de Dieu, si bien que toute personne qui le contemple se trouve plongée par Dieu dans l'amour. Considérons le rêve en détail:

Quant la nuit déchira la poche de son musc afin de le verser sur la robe du jour, un ange vint montrer à Ziyâd en son sommeil un splendide parterre illuminant le monde; son étendue, grâce à la hauteur de ses arbres, était gaie tout autant que le cœur des heureux; toute fleur épanouie paraissait un jardin; tout pétale de rose était comme un flambeau. Un pavillon céleste en émaux de couleur comme $\mathrm{si}^{5}$ un œil clairvoyant était dans chaque herbage; l'émeraude n'était pas plus verte que lui, et quant à sa splendeur, elle était sans limites. Les roses épanouies présentaient une coupe, et le rossignol ivre entonnait sa chanson; quand les musiciens maniaient leur archet, les ramiers répondaient par leurs roucoulements. (Rûzbehân 1985, $\$ 270$ )

La mention de l'ange vise à attester la pertinence oraculaire du rêve et entend garantir qu'il ne sort pas de la fabrique de quelque démon qui entreprend une fallacieuse apologie de la passion amoureuse ${ }^{6}$. Il est possible d'ajouter une troisième raison, que l'auteur ne pouvait sans doute pas avoir en vue: comme selon l'oniromancie arabe la vision d'un ange est de bon augure ${ }^{7}$, elle laisse prévoir pour Ziyâd un accomplissement personnel à l'image de l'apothéose de son ami.

Deuxième trait: luminosité et irradiation. Voici la part obscure de l'expérience amoureuse dépassée. Absence et séparation se servaient de la métaphore de la nuit. Mais n'en serait-il pas de même de l'union, comme on le voit chez Novalis? De fait, il n'est de clairière que par la distance. Or le poème insistera sur l'identité des amants. Laisse-t-il place à une dualitude au sein même de l'identité ? On peut être de cet avis. À moins qu'il ne faille plutôt remonter à la source de l'extrême clarté en deçà même de la rencontre

5. J'ai modifié la traduction qui porte ici: tel.

6. En effet, d'après une opinion rapportée par Mas'ûdî (1841-1877, 359), les songes proviennent soit d'anges soit de démons.

7. Voir Ibn Sîrîn $(1993,93)$ et Lory $(2003,145-149)$. 
amoureuse et qui est sa condition de possibilité, le 'ishq qui est feu. Or nous savons que ce ne sont pas les amants qui boutent le feu. Il leur revient seulement de l'alimenter, étant pour lui ce que sont les attributs par rapport à l'essence. C'est pourquoi ils n'acquerront l'immortalité que pour avoir été embrasés. Autrement dit, si l'être est 'ishq, seuls existent et «surexistent » les amants. L'errance de l'un et le confinement de l'autre apparaissent alors comme les deux formes, visible et invisible, d'une même déterritorialisation qui s'exprime désormais dans une troisième forme, extrêmement épurée: l'extraposition au Paradis, autrement dit une déterritorialisation qui se présente sous l'aspect d'une territorialité absolue, métaphore de la participation à la substance du 'ishq. On remarque que la lumière se répand sur le monde (au singulier) et pas seulement sur l'espace édénique (le monde supérieur). La preuve: cette même lumière envahit le rêve de Ziyâd. C'est la révélation qui descend en lui, ce n'est pas lui qui introduit dans le Paradis l'œil visionnaire. Quand bien même imperceptible pour beaucoup, le rayonnement se diffuse partout.

Quant à la dominante verte, elle connote certes la vie permanente, mais aussi une détermination spécifique de la lumière spirituelle comme état, car selon Najm al-dîn Kubrâ (1993, 153), exact contemporain de Nizâmî, l'éclosion smaragdine correspond à la vitalité du cœur où se recueillent toutes les significations et se résolvent les dissonances. De là procède, dans le poème, l'harmonie entre le chant de la nature (rossignols et ramiers) et le jeu des musiciens. Le poème poursuit:

À l'ombre des rosiers, brillant comme soleil, un trône se trouvait posé au bord de l'onde; ce trône était orné de tapis de brocart qui étaient aussi beaux que ceux du Paradis. Là deux anges bénis de Dieu, dans leur beauté siégeaient sur ce trône de liesse. Tous deux resplendissaient des pieds jusqu'à la tête, pompeusement parés, comme houri dans sa robe. En présence du frais printemps, la coupe en main, chacun d'eux racontait à l'autre son histoire; tantôt mettant leurs lèvres à celle de la coupe, et tantôt unissant pour un baiser leurs lèvres; de temps en temps tous deux échangeaient des paroles ou bien ils s'endormaient au gré de leur désir. (Massé 1997, 76-77)

L'angélométamorphose ${ }^{8}$ des amants qui ont brûlé leur vieille tunique est la gloire de la volupté pure qui circule dans les hauts lieux. La nature de leur transfiguration atteste qu'ils sont devenus de part en part lumière. Or, translucides, ils ne laissent voir que le feu.

8. Nîzâmî croit que l'homme peut devenir ange (Les Sept Portraits, 41). 
Il est remarquable que sur le trône ne siège pas seulement Majnûn. C'est le couple comme tel qui règne. Ce qui était désir aveugle dans le sensible advient comme étreinte clairvoyante dans le supra-sensible. Majnûn fut deux (l'amant et l'image de l'aimée) qui pensa l'union moyennant la sentence d'identification tout de même que Majnûn et Laylâ sont aujourd'hui un tout en restant deux. Unité et dualité se compénètrent dans l'expérience du baiser qui n'est pas unique. La surexistence édénique comble absolument, et cela apparaît avec d'autant plus d'évidence si on se rappelle quel souhait modeste Awhadi imaginera pour Majnûn (v. 269-270; Massé 1997, 249):

Il ne me reste qu'un désir : baiser la main de cette amie,

Le jour qu'au son de la trompette du sépulcre je sortirai.

Pas moins que le baiser l'ivresse semblait interdite aux amants tant qu'ils suivaient le cours du monde sublunaire. Or voici que par-delà le clivage du licite et de l'illicite, le vin est répandu à profusion dans le Paradis de l'Islam (comme Majnûn le devine, le vin de l'amour ne peut être goûté qu'au Paradis). Des fleuves de vin irriguent le Jardin (Coran 47,15) et dans les coupes circule le breuvage convoité (Coran 83,25-26). Métaphore de l'extase, l'ébriété couvre l'expérience de la subjugation et de la perte de conscience sous l'effet de la jonction, ou même simplement de la passion. Boire de la coupe de l'amour divin au point de s'enivrer devient une formule très tôt banale. On la trouve déjà sous la plume de Yahyâ Ibn Ma'âdh al-Râzî, objet de l'acerbe raillerie de Bistâmî pour s'être trop vite altéré. Certains font suivre l'ivresse par la sobriété, degré supérieur (Kalâbâdhî 1960, ch. 55). D'autres les identifient. La sobriété peut passer pour une forme supérieure de 'ishq, puisque ce dernier se dissimule dès lors que l'amant est dans la perfection de l'amour. Il semble qu'ici prévale l'idée d'une extase dialogante qui suit trois phases: coupe, baiser, paroles. Cellesci reconduisent les amants à leurs ipséités respectives, puisqu'elles rapportent l'histoire de chacun. Mais comme sous cette entité fallacieuse se déploie l'être comme 'ishq, l'immanence de chacun à son propre état (passion révélée et passion dissimulée) se réfléchit dans l'unique expérience de la passion, unicité dont le caractère ineffable restitue les amants à l'ivresse, et derechef à l'étreinte. Il est fait mention d'un sommeil qui rythme l'existence nouvelle. Serait-ce, comme le baiser ou l'ivresse, une métaphore euphémisante du commerce charnel ? Il n'est pas improbable que souvent l'idéalisation de la Dame recouvre un effroi devant la sexualité féminine. Le rêve 
semble ici hésiter entre deux positions: celle de l'épreuve, dans la version aussi bien provençale que 'udhrite de l'amour courtois, qui autorise une visite de l'amoureux dans l'alcôve de l'aimée à condition de ne pas passer à l'acte; et celle de la traduction physique de l'inclination. C'est tout le jeu de la censure, tournée en bienséance, que de suggérer l'accomplissement total et l'apaisement après qu'on ait longtemps enduré l'antagonisme des pulsions. C'est le destin du rêveur, Ziyâd, que de ne pouvoir se représenter la possession de l'aimée qu'en dehors de lui-même, dans le médium fantasmé du couple paradisiaque. Dans l'étreinte elle-même règne la nuit, mais la vision de l'étreinte exige la distance, et donc la pleine lumière. Les ténèbres sont bannies du Paradis onirique pour la bonne raison qu'il reste offert à des yeux qui ne cillent point. Ici la question se pose: qu'est-ce que le Paradis? Il ne consiste pas dans la contemplation de Dieu, à moins que les amants puissent passer l'un pour l'autre pour Dieu, mais alors lequel ? Le Dieu véritable exige une translation du reflet à l'original. Ce n'est plus le lieu d'adorer l'Un sous la figure de l'aimée. Que si l'on y persiste, il y a crainte que les amants ne sombrent dans l'idolâtrie. À moins qu'ils aient obtenu licence de ne pas s'adonner aux plaisirs sensuels consentis aux élus afin qu'ils puissent se consacrer l'un à l'autre. On se souvient que Laylâ était le paradis de Majnûn.

Le poème fait ici intervenir un personnage inattendu: "Et debout, un vieillard, tout à ses dévotions, avait posé la tête au sommet de leur trône; à tout instant il remuait des pièces d'or et les répandait sur les têtes des amants. En secret, le bédouin qui contemplait ce rêve, posa ces questions à ce vieillard céleste: "Ces deux êtres si beaux qui tiennent une coupe, quels noms portent-ils donc au jardin des élus? Dans le séjour du Paradis ils ont pris place; mais d'où vient donc qu'ils ont obtenu ce haut rang?" Aussitôt le vieillard éprouvé par le temps lui répondit, parlant en sa langue muette :

Ces amants qui sont deux ne font qu'un être unique; c'est pour l'éternité qu'ils restent compagnons. L'un est maître absolu sur son cheval arabe; l'autre par sa douceur est la lune des belles; or Majnûn est le nom de celui qui est roi; et celle qui est lune, on l'a nommée Laylâ. Ils ressemblaient à deux rubis encore intacts dans un coffret d'amour et de fidélité; mais dans ce monde, ils n'ont pas connu le repos, ils n'y obtinrent pas l'objet de leurs désirs. Ils ne subiront plus, ici, d'autre souffrance que de rester ainsi réunis pour toujours. Ceux qui n'ont pas mangé les fruits de ce bas monde, c'est ainsi qu'en ce monde, ils redressent la tête. Celui qui sur la terre est la proie 
du chagrin, telle est la joie qu'il doit trouver dans l'au-delà. (Massé 1997, 76-77)

Le vieillard intervient comme révélateur de l'identité des amants transfigurés. La question montre déjà que Ziyâd ne les a pas reconnus tant leur transformation fut grande et leur union paradisiaque extraordinaire. Il ne lui est pas permis de les interroger car rien ne peut ni ne doit distraire les amants de leur adhésion à la substance de la passion. Le sage chenu (une sorte de double du poète) qui leur rend un hommage appuyé le fera donc à leur place. Rien ne dénote pourtant une divinisation des deux personnages car les gestes sont de ceux qui honorent les rois ou les mariés de haute condition.

Puis la morale du rêve: le Paradis induit la permutation des conditions terrestres et l'accomplissement des désirs ${ }^{9}$. Pour cela, une condition: l'ascèse qui est fidélité dans l'univers de la séparation. "Deux rubis encore intacts dans un coffret d'amour et de fidélité »... Nous savons que tel n'est pas le cas pour Laylâ dans la légende arabe, sinon seulement peut-être eu égard à son sentiment inavoué. Or Nizâmî innove et transmet à ses imitateurs l'idée de la résistance victorieuse de la jeune femme, face aux avances légitimes de son mari. Est écarté tout paradigme du rival heureux. Si les rites de Vénus n'ont pas été accomplis, la virginité des deux amants incorpore le sens de la fidélité et justifie leur union paradisiaque.

La question se pose du destin réservé à l'infortuné époux que Nizâmî a fait non moins tragiquement amoureux de Laylâ, laquelle n'éprouve qu'horreur pour ses empressements. Froideur et dédain étant son lot, Ibn Salâm, contraint à la continence en dépit de la proximité et du droit, voit sa situation se compliquer par rapport aux amants déjà unis par la passion, ou plutôt en elle qui relève de l'être et non du monde. Si leur liesse paradisiaque n'est qu'une projection leur permettant de contempler une réalité indécelable même à leurs yeux, alors c'est en l'époux pitoyable, adorateur transi et victime humiliée d'un Noli me tangere quotidien et sans complaisance $^{10}$, que l'ascèse est paradoxale: il est du monde tout en n'étant pas du monde; il est uni tout en ne l'étant pas; c'est lui qui, comblé de feux, porte tout le poids de l'amour pur comme amour damné. Signe cette

9. On peut comparer avec le traité de Swedenborg sur les Charmes de l'amour conjugal qui porte sur les unions célestes.

10. Il y a un syndrome nizâmien de la femme qui se refuse à celui qui la désire ou qui sème sur son parcours des embûches létales. Voir dans les Sept portraits les histoires des Dômes jaune et rouge. 
damnation son absence totale de la scène qui nous occupe. La loi, révélée ou sociale, perd son emprise, elle qui, dans Les Affinités électives de Gœthe, réclamait implacablement son dû en dépit de la nature. N'avoir su ou voulu consommer le mariage ne donne aucun droit de compensation, bien au contraire. L'accidentel est implacablement éliminé au pays de l'essence. Rien d'étonnant à cela dans un paradis de l'amour, et s'il est avéré que l'être est 'ishq, ce dernier ne saurait être constitué par des préceptes socioreligieux et encore moins suscité par leur transgression. Le fou, comme dit Jâmî après Rûmî (Jâmî 1993, 211), n'est pas justiciable de la loi ${ }^{11}$. L'amour courtois, en Occident, s'acclimate sur un tout autre terrain où l'essence ne se conçoit pas sans la donnée de l'appartenance à autrui. L'infidélité s'y conçoit à deux sens, quoique non pas selon la même valence. La Dame se dédie à deux hommes, mais dans l'un des deux cas, c'est pour observer son devoir, si bien que l'amant ne saurait être affligé par les étreintes conjugales. Voici l'explication qu'en donne l'Anonyme d'Erfurt: «L'amour entre l'époux et l'épouse est comme une dette naturelle, tandis que l'amour entre deux amants est gratuit et naît d'une volonté libre. Ces deux amours n'ont rien de commun, et l'amour conjugal en acquittant sa dette ne porte aucun préjudice à l'amour gratuit » (Traité de l'amour parfait, 67). Dans le roman persan, une forme d'amour a été avantagée par rapport à l'autre. Il revient à l'épouse de rompre tout lien, dès lors que la Parque a coupé le fil. Il aurait été plus difficile de juger la Laylâ de la légende arabe qui devait livrer son corps à l'un tandis qu'elle se disait éprise de l'autre dans le secret, situation qui est comme une lointaine réminiscence de la dichotomie de l'amour divin et du devoir sexuel qu'aurait vécue Râbi'a sans affres, dans une légende qui la donne pour mariée. Il est raconté que chaque soir elle se parfumait à l'intention de son mari et se livrait à lui s'il avait le désir d'elle. Son cœur, lui, appartenait à un Autre. Le poème suivant lui est attribué:

J'ai fait de Toi celui qui m'entretient dans le cœur

Tandis que j'ai rendu licite (abahtu) mon corps à qui se veut

Mon hôte. Du compagnon mon corps se rend familier,

Mais l'aimé de mon cœur est mon familier dans l'intime ${ }^{12}$.

11. Leylî u Majnûn, v. 1368. Je suis l'édition procurée au tome II de Haft Owrang, Téhéran, 1997.

12. Cité avec l'anecdote précédente dans Munâwî (1999, 288). 
La situation est volontairement tragique chez Nizâmî (et Jâmî). C’est que la Dame qui impose chasteté à tous prend le dessus sur les hommes et ne renonce au plaisir que pour se confirmer dans sa semi-divinité d'être universellement adulé qui propose une féminité qui n'est plus l'objet d'une appétence grossière, mais d'un amour sublimé dans les hautes régions où prennent naissance et agissent les anges ${ }^{13}$. Son amour pur subvertit l'ordre social en cet empire absolu qu'elle exerce sur son seigneur (j'entends son mari, le baal...). Mais c'est à se demander si l'amour pur hyperbolique du mari, en sa passivité, n'est pas davantage subversif dès lors qu'il se complaît dans le renversement sans faire retentir, à l'inverse de Majnûn, toute la nature de ses plaintes. Quoique la condition lui soit imposée, sa passivité ne plaide-t-elle pas contre le droit et les convenances ? Bien que Laylâ puisse admettre que l'amour suprême ne trouve pas son accomplissement sur terre, elle ne va pas jusqu'à reculer devant la mort, au motif qu'elle aurait des devoirs à remplir en ce monde. C'est qu'elle a réussi à être libre de toute maternité.

Au portrait d'un Majnûn révolté que nous propose Rûmî, un contrepoids est suggéré: l'amant se parfait dans l'extinction. Il est symptomatique, par le malaise que fait naître le cas d'un époux frappé de passivité, que Hâtefî̀ ${ }^{14}$, neveu de Jâmî, modifie la donne en faisant Ibn Salâm répudier «mâlement» la beauté rétive.

Deux issues sont entrevues, paradisiaque et infernale. La damnation de l'usurpateur se signale comme un paradis inversé. C'est que Laylâ est paradis. Un vieillard qui servait de messager entre Majnûn et Laylâ décrit son habitat avec des traits et des couleurs empruntés au lieu céleste, et sa belle occupante est comparée à une houri. On se rappelle le vers de Rûmî:

Partout où un Joseph, beau comme la lune, se trouve,

C'est le Paradis, quand bien même ce serait le fond d'un puits (Mathnawî II, v. 3811)

Mais n'est-ce pas à condition d'être jeté avec Joseph dans la fosse? C'est ce même paradis qui torture lorsqu'il ne désaltère pas. Pire encore,

13. À cet égard, le contraste est grand entre le paradigme de Laylâ et celui de Wîs, laquelle, si elle frappe d'impuissance son mari, prend amant qu'elle élève par les fascinations de la beauté jusqu'au mystère de l'union d'amour comme abandon à l'aimée.

14. Le poète ne manque pas non plus de présenter Majnûn comme un mystique parvenu à la perfection par la voie de la passion amoureuse, après avoir lavé ses fautes dans les pleurs... 
Laylâ est une lune aux yeux de son époux; en revanche ce dernier lui paraît un dragon ravisseur. S'avise-t-on assez que des trois amoureux, c'est Ibn Salâm qui le premier est conduit sans esquive possible dans la fosse? Ravagé par d'inquiétantes et pathogènes consolations, miné par sa mélancolie de tiers exclu ${ }^{15}$, acculé au désespoir, il n'a éprouvé de la passion que le versant dolent, non celui de la joie. Il reste à l'entrecroisement de la possession et de la béatitude sans vivre pleinement l'une ou l'autre. Certes, il entre en jouissance de son ipséité lors même qu'il souffre, puisqu'il se reçoit alors de la haine de l'aimée. De l'être qui est 'ishq il fait l'expérience, à ceci près que la permutation tourne encore plus en sa défaveur, puisqu'il ne lui est plus donné de se repaître de la beauté de l'intouchable. C'est que l'amour continent se décline suivant deux modalités, hyperbolique et simple: amour pur damné et amour pur partagé. Tous deux procèdent du 'ish q et vivent de chasteté. Mais le second doit d'être pur à la séparation qui est un fait du monde, tandis que le premier doit de ne l'être pas moins à la non-réciprocité qui n'est pas un fait du monde, mais bien un fait de la vie du 'ish $q$ dans ses attributs. À cet égard, la scène paradisiaque sert à discriminer ces deux modalités, car tant que le Majnûn de Nizâmî était en vie, il n'avait nulle assurance de réciprocité et de fidélité, à l'instar de son modèle arabe. Le rêve vaut élucidation. Sitôt les paramètres du monde suspendus (ce que le rêve produit aussi bien que l'élévation à la condition béatifique), la vérité des amants se traduit en vérité de la jonction, tandis que le damné d'amour s'éclipse à tout jamais, comme s'il n'avait été qu'un simple opposant (au sens de Propp) dont le rôle s'achève et l'existence s'estompe dès lors qu'un terme est mis à l'action — lorsque le temps, dira-t-on avec le Parsifal de Wagner, se change en espace. Corporellement intacts par l'ascèse de la chasteté, les trépassés d'amour profitent de la magie transvaluatrice de la puissance onirique pour s'élever jusqu'à l'intemporel sol des retrouvailles. Seul le rêve comporte, par-delà tout mirage, une signification assimilable par l'imaginaire poétique et qui prononce sur le 'ish $q$ un verdict d'éternité. Il n'est pas vrai, selon Nizâmi, que ce spasme disparaisse avec la mise en bière ou que sa lame meurtrière emporte tout, car il fait le fond de l'être. Il a beau déposer l'individu autonome, il ne fait que reconduire l'humain, par les chemins de la constante ardeur, au vif absolu. Ce vif absolu,

15. On peut ajouter, bien que l'idée ne corresponde pas à l'intention de Nizâmî, que la mélancolie peut être provoquée par la continence selon Abû Baqr al-Râzî (cité par Ibn al-Jawzî 1994, 292). Ibn Qayyim al-Jawziyya, qui fait du 'ish q une modalité de la mélancolie, retient que les rapports sexuels en délivrent (1956, 137). 
le rêve nous le donne dans la guise d'une jonction. L'arbre unique du rêve de Majnûn se divise pour produire l'image de l'entrelacement des branches de l'essentiel désir, de l'arbre mercuriel ou lunisolaire de la tradition alchimique (Jung 1971, 404 et 450), ou de deux arbres, ceux qui obombrent l'unique tombe des deux amants. La totalisation posthume qui fait le thème du poème final de Majnûn et Laylâ advient de ce côté-ci de l'existence dans l'autre grand roman de Nizâmî, Khosrow et Chîrîn.

\section{Références}

ABÛ AL-Qâsim (1923), Kitâb al-'ilm al-muktasab fî zirâ'at al-dhahab, trad. E. Holmyard, Paris, Geuthner.

AL-QushaYrî (1996), Risâlat, Beyrouth, Dâr al-Jîl, p. 322.

Hafiz, Dîvan-e Hâfez, Téhéran.

Hatem, J. (2000), Mal d'amour et joie de la poésie chez Majnoun Layla et Jacques Jasmin, Agen, Quesseveur.

- (2005), Hallâj et le Christ, Paris, L'Harmattan.

- (2006), Satan, monothéiste absolu selon Gœethe et Hallâj, Paris, Éditions du Cygne. (2008), La Poésie de l'extase amoureuse, Paris, Orizon.

IBN AL-JaWZî (1994), Talbîs Iblîs, Beyrouth, Dâr al-Nadwat al-jadîdat.

IBN 'ARABî (1961), Risâlat al-ittihâd al-kawnî fî hadrat al-ishhâd al-'aynî bi mahdar al-shajara al-insâniyya wa-l-tuyûr al-arba'a al-rûhâniyya, Le Caire.

IbN QAyYim Al-JaWZIYYA (1956), Rawdat al-mubibbîn, Le Caire.

IBN SIRIN (1993), Le Grand Livre de l'interprétation des rêves, trad. Y. Seddik, Beyrouth, Al-Bouraq.

Jung, H. (1971), Les Racines de la conscience, Paris, Buchet/Chastel.

KALABADHI (1960), Kitâb al-ta'arruf li madhhab ahl al-tasawwuf, Le Caire.

LORY, P. (2001), «Les Paradoxes mystiques: l'exemple de Shiblî », dans L'Orient des dieux, I, Beyrouth, Université Saint-Joseph. Michel.

Masse, H. (1997), Anthologie persane, Paris, Payot. 
MAS'ÛDî (1841-1877), Les Prairies d'or, t. 3, trad. fr., Paris.

Mullâ SADrÂ (1966), Al-Shawâhid al-Rubûbiyyat, Mashhad.

MunAwi (1999), Al-Kawâkib al-durriyyat fî tarâjim al-sâdat al-sûfiyyat, t. 1, Beyrouth, Dâr Sâdir.

Najm Al-Dîn Kubrâ (1993), Fawẩih al-jamâl wa fawâtih al-jalâl, Koweit, Dâr Su'âd al-Sabbâh.

NizÂMî, Leylî u Majnûn, Shîrâz.

NizÂMî (1987), Le Trésor des secrets, trad. D. Mortazavi, Paris, DDB.

PERnety, A.-J. (1758), Dictionnaire mytho-hermétique, Paris.

RûMî (1990), Mathnawî (La Quête de l'Absolu), tr. E. de Vitray Meyerovitch et D. Mortazavi, Monaco, Éd. du Rocher.

- (1993), Odes mystiques, trad. E. de Vitray-Meyerovitch et M. Mokri, Paris, Seuil.

RûZBehân (1985), Le Jasmin des fidèles d'amour, tr. H. Corbin, Paris, Lagrasse.

SAFÂ, Z. (1987), Anthologie de la poésie persane, Paris, Gallimard.

SARRAJ (1960), Kitâb al-lumâ' fi-l-tasawwuf, Le Caire.

\section{Résumé}

Après avoir acquis son titre de référence des amants, la figure de Majnûn Laylâ a été mobilisée par la mystique pour exprimer l'amour pur, à savoir l'union à Dieu en dépit de l'absence. À l'instar du Majnûn de la légende arabe, qui s'est enfoncé sans recours et jusqu'à la folie dans l'amour désespéré, celui de la mystique voue à Dieu une dilection sans bénéfice. Dans le roman que Nizâmî lui consacre, deux rêves, l'un du personnage principal, l'autre d'un compagnon, viennent rétablir l'équilibre. Dans le premier, de teneur subjective, l'amant est consacré roi, ce qui est de nature à concevoir un accomplissement au sein même de l'absence; dans le second, la réunion des amants dans la mort redéfinit le paradis comme le lieu de l'union.

\section{Abstract}

After having become the point of reference for lovers, the character of Majnoun Laylâ was also mobilized by mysticism to express pure love, mea- 
ning union to God despite the absence. Just as the Majnoun of the Arab legend hopelessly sinks into desperate love until madness ensues, the mystic Majnoun's love of God does not involve material returns. In Nizami's novel about Majnoun, two dreams, by the main character and his companion, restore the balance. The first, quite subjective, sees the lover elected king, which permits self-realization in the very midst of absence; in the second one, the lovers are reunited in death redefining paradise as the place of the union. 